\title{
Concept for completeness checking of joined structures exemplified on rail vehicle car body shells
}

\author{
Uwe Jurdeczka \\ ALSTOM Transport Deutschland GmbH, Linke-Hofmann-Busch-Straße 1, 38239 Salzgitter, Germany \\ Correspondence to: Uwe Jurdeczka (uwe.jurdeczka@transport.alstom.com)
}

Received: 31 August 2016 - Revised: 31 December 2016 - Accepted: 4 January 2017 - Published: 31 January 2017

\begin{abstract}
Completeness checking of constructional steel structures with several hundred weld-on parts (mountings, such as car body shells/chassis for rail vehicles, for example) still represents a great challenge. It has been performed manually so far. By the use of 3-D scanners, it is possible to obtain sufficiently comprehensive information about the actual configuration (as-is state) of the constructional steel structure to be checked. For this purpose, the laser beam geometry must be contemplated. By using an adapted algorithm for post-processing of the resulting point clouds, difference figures are superimposed over the respective 3-D model as the target state. Therefore, an algorithm was adapted and applied here. The difference figures are useful for signalling the possible deviations and the missing parts, at least, however, suspect points. Thus, the disadvantages of the exclusively manual completeness checking practiced so far, i.e. tiredness and slowness of the inspector, can largely be avoided. The 3-D model-based inspection procedure allows one to handle the variation in the target state with just a few volumes of equal configuration (e.g. less than 10 trains with equal configuration). There is a general problem that the tolerance for the structure is in the same range as the size of the mountings. It is possible to solve this problem by dividing the test item as well as the point cloud into areas. Superposing the 3-D model by point clouds and calculating the difference figures will be performed area by area.
\end{abstract}

\section{Introduction and motivation}

Completeness checking of constructional steel structures with several hundred mounted parts (such as car body shells/chassis for rail vehicles) represents a great challenge. Cycle time and constructional variant diversity with a low quantity of completely identical car body shells represent the motivating general conditions. This variant diversity is managed in production by MES, Manufacturing Execution Systems. The production-accompanying checks also require attention. The variant diversity is based on the type of mounted parts, their number and their positions. About 300 mounted parts (holders, lugs, earth connectors or the like) are to be checked for completeness within the cycle time per shift (approx. $8 \mathrm{~h}$ ). Although production-accompanying completeness checking has long been performed, with the test personnel having been provided with many aids (e.g. from checklists and sector classification of the test objects to inspection/break time regimes), this completeness checking has largely remained a manual visual inspection until now. Oc- casional check slip leads to additional effort in the following operations. The development of sensors and image processing systems has provided other aids to relieve the test personnel and to improve the fault rate of the manual visual inspection.

\section{Preliminary investigations}

The development of equipment-based completeness checking is proceeding. The current state, regarding bigger constructions than cars, is described (i.a. Tarih, 2016). Application reports of first developments to equipment- and modelbased completeness checking - for smaller inspection volumes than described here - were already presented (Berndt and Warnemünde, 2012; Kelch, 2015; Stemmer, 2015; Demant et al., 2012; Berndt and Sauer, 2014). Störing and Zimmermann showed measurement and even completeness checking for bigger attributes (Störig and Zimmermann, 2015). This development in completeness checking is added 
by another way to prevent missing parts, namely instructions to the assembly staff and to the inspectors, where target positions of weld-on parts (mountings) are indicated by laser light on the mounting position or by monitor in a computer-aided design (CAD) data set (Drechsel, 2016; Extend3d $\mathrm{GmbH}$, 2016; Optimum datamanagement solutions GmbH, 2016).

\subsection{Conclusions of preliminary investigations}

The known approaches for completeness checking should therefore be continued to describe a test procedure that gets along with a reasonable use of equipment (investment of a man year) and that does not affect the given cycle time (one shift, $8 \mathrm{~h}$ ). Model-based checks now promise the possibility of considering the variant diversity of design and production by means of easily adaptable test plans and criteria lists.

The generation of difference figures from the comparison between 3-D CAD data and actual 3-D data has already been described, without having yet considered the allowed manufacturing tolerances. Thus, a deviation in length of the real steel construction from the data set, for example, is indicated in the difference figure. However, such a deviation would not necessarily be a fault but a variation in production evaluated as still in order. Thus, a whole side of a cubic steel construction (e.g. the side wall of a rail vehicle) might be presented as missing/deviant although there is no fault but that the tolerance is used up to nominal size (so-called error of the second kind).

Difference figures result from superposition of a 3-D model (CAD file) with a point cloud or the mesh calculated therefrom. It finds the relation between points/mesh elements and their nearest neighbours in the corresponding areas of the 3-D model. The value of the distance is used for colour coding.

The challenging task is to check for the presence of mounted parts that might be considerably smaller than the manufacturing tolerances of the big structure they are mounted to.

The available equipment classes and the analysing methods should also be modified and adapted to

- the capture of the as-is state of the test object (e.g. point cloud, 3-D scan) and

- the analysing algorithm for signalling deviations and solving with the general problem with two different tolerance fields (described later on).

Optical methods seem to be suitable because of the elongation of the test objects since a large number of test object data can thus be obtained within a short period of time.

A terrestrial 3-D laser scanner (TLS, also called HDS high-definition surveying) seems to be congenial. One of the reasons is that the results were displayed as $x, y$, and $z$ coordinates in absolute dimensions. This means that there is

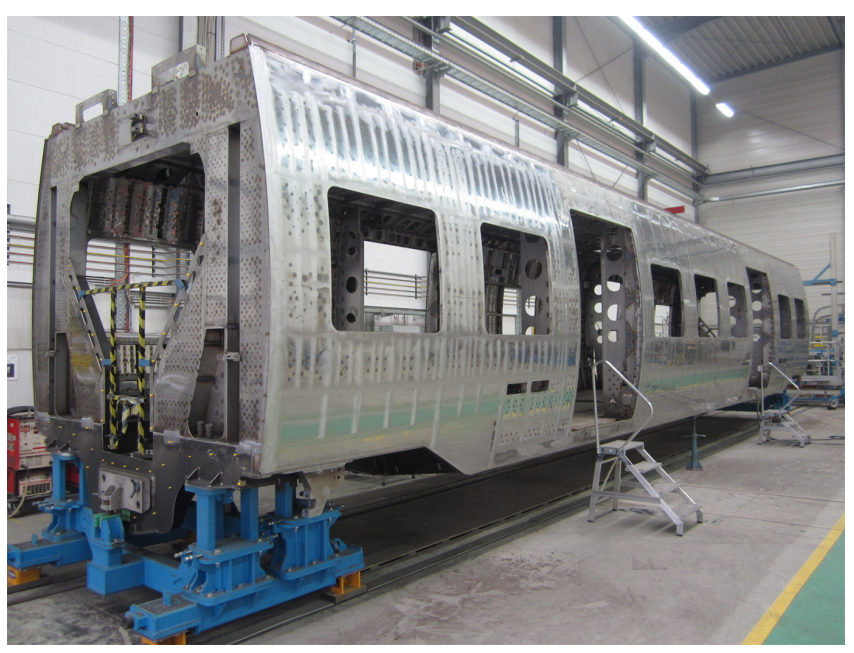

Figure 1. A car body shell as an example of a test object in a test rig.

no additional calculation necessary, based on the principle of spatial image triangulation calculation. Photogrammetry with a hand-held camera is an example where such spatial image triangulation calculation would be necessary. Nevertheless, the calibration of the TLS is possible and described (Wunderlich et al., 2013).

Two different principles are applied to distance measurement in equipment class Terrestrial Laser Scanner.

\section{a. Time-of-flight (TOF)}

A light pulse is temporarily sent from the laser scanner, passes the distance to the object and back and is then received again by the laser scanner with a time delay proportional to the distance.

\section{b. Phase shift}

In the phase shift process, modulated laser light is continuously emitted and the phase shift of the modulation wave of the reflected light is permanently determined. The phase shift is proportional to the length of a remaining part of the total object distance. This remaining part can be maximally as long as the wavelength of the modulation wave. Thus, a portion of the total distance regularly remains indefinite, which corresponds to an integral multiple of the modulation wavelength. This so-called ambiguity problem is solved by the use of different modulation waves (Reiterer et al., 2015).

Available software program packages were used to perform subroutines of the described algorithm (Jurdeczka, 2016a). Preliminary investigations have shown that the handling possibility of very large point clouds for the issue of difference figures varies indeed. With PolyWorks, provided by company Duwe-3d AG, Lindau, with SpatialAnalyzer, provided by company VMT GmbH, Bruchsal, and with 3- 
D Reshaper, provided by company Technodigit, very satisfying results could be achieved. These statements on the software program packages could be obtained in individual test phases.

\section{Description of concept}

The concept presented here demonstrates a test system for the completeness checking of mounted parts. It is possible to obtain information about the actual state of the test object by 3-D scanning. And in a second step, by computing difference figures, predications about the completeness of the mounted parts were given.

\subsection{Measurement task}

The measurement task is embedded in the inspection task to perform a completeness check. As far as is possible, to get a point cloud from a 3-D scan, the as-is state of the car body shell, inside, is represented by this point cloud.

This point cloud is to be processed to a difference figure. And in the difference figure, there are predications about the completeness of the mountings inside the car body shell.

\subsection{Measurement systems}

In contrast to the methods already described, a TLS is used here for data acquisition (3-D scan, point cloud). Besides the result being given in absolute dimensions, measurement speed, a comparatively easy operation and manageable point clouds are the advantages resulting from the use of TLS/HDS for getting the test object image.

The equipment parameters are adapted to the test object so that the quality of the scanned point cloud (point density, intensity, included and largely automatically recognized targets) is at least sufficient for the further analysis.

Tests have shown how the equipment parameters can be adapted to the test object (glossy surfaces, a comparatively narrow focus area for the equipment class). For this purpose, various wavelengths of the scanning laser beams, different beam diameters and also equipment-specific settings for the evaluation of the reflecting beam are to be considered.

For these process studies, Leica Geosystems (Leica Geosystems GmbH Vertrieb, Düsseldorf) provided the ScanStation P40, a state-of-the-art time-of-flight scanner (TOF scanner). Thus, suitability for surfaces and geometries could be confirmed in a series of tests. Figure 2 shows the obtained point cloud at one of the five sensor positions.

In another series of tests this suitability was also proved for phase shift scanners by means of the Surphaser 100HSXSR provided by LIMESS Messtechnik und Software GmbH, Krefeld.

Both types of scanners (TOF and phase shift) were usually used in ranges of up to $270 \mathrm{~m}$. But they can be used for the inspection task here, provided that the set-up is appropriate

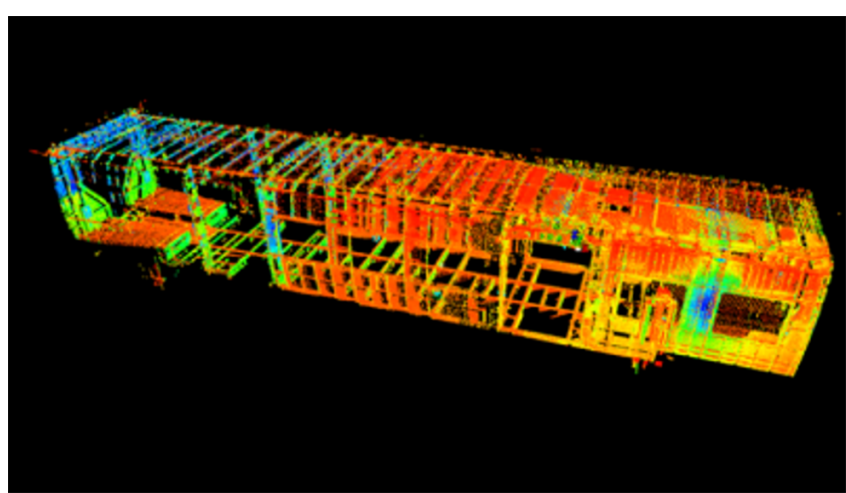

Figure 2. Example of a point cloud. The point cloud is shown as a scanning result of one sensor position (of a total of five positions for this type of car body shell); the colour marking demonstrates the intensity of reflection (remission).

and the configuration is adapted to the measurement range, especially closest measurable distance and effective range.

\subsection{Measurement strategy}

Due to the size of the test object, it is advantageous that the preparation of samples (cleaning, matting or the like) can be omitted for the accuracies pursued here. The partially strongly reflecting cold-rolled steel surfaces pose high requirements to the parameter selection for intensity and evaluation of software-based filters. Pillars inside lead to shadowing. The shadowing of criteria (undercutting) can be considered by multi-sensor systems or, as practiced here, by sensor movement within the test object. A typical car body shell of a regional train can be scanned inside using five or six different sensor positions to avoid shadowing.

The analysis of the scanned point cloud in software algorithms cannot be completed during the scanning time, but is continued up to several hours. The time for post-processing is a function of sensor positions, point density during scanning and parameters for meshing, and can take long, up to hours. Nevertheless, compared with the completeness checking of up to $8 \mathrm{~h}$ practiced so far, there is a great potential for saving time for completeness checking in general.

The mounted parts are of different sizes. The smaller ones have typical dimensions of $15 \mathrm{~mm} \times 25 \mathrm{~mm} \times 1 \mathrm{~mm}$. Several 3 -D scanner probes should therefore be applied to these lugs to provide a sufficient number of actual points for the subsequent analysing algorithm. At a scanning distance of about $2 \mathrm{~m}$, scan densities of $6 \mathrm{~mm} @ 10 \mathrm{~m}$ (adjustable on the 3D scanners) lead to point densities in scanning of approx. $1.2 \mathrm{~mm}$, which turned out to be sufficient for the interior of the car body shell. Figure 3 presents a single point cloud from one sensor position, scanned with Surphaser 100HSX-SR. 
Table 1. Data of beam geometry and measurement principles of various terrestrial laser scanners.

\begin{tabular}{lll}
\hline & ScanStation P40 & Surphaser 100HSX-SR \\
\hline Beam diameter at exit & $\leq 3.5 \mathrm{~mm}(\mathrm{FWHM})$ & $1 \mathrm{~mm}$ \\
Beam angle & $<0.23 \mathrm{mrad}(\mathrm{FWHM}$, full angle) & $\begin{array}{l}\text { Not given, but the beam diameter is } \\
1 \mathrm{~mm} \text { in the measurement range. }\end{array}$ \\
Wavelength & $\begin{array}{l}1550 \mathrm{~nm} \text { (invisible) } / 658 \mathrm{~nm} \text { (visible) } \\
685 \mathrm{~nm}\end{array}$ \\
Distance measurement principle & $\begin{array}{l}\text { Time-of-flight (TOF) enhanced by } \\
\text { waveform digitizing }\end{array}$ & Phase shift \\
Recommended measurement range & $0.4-270 \mathrm{~m}$ & $1-7 \mathrm{~m}$ \\
\hline
\end{tabular}

Table 2. Time required for scanning and checking (scanning and setting-up) based on a capture at a point density of $6 \mathrm{~mm} @ 10 \mathrm{~m}$ for various numbers of sensor positions.

\begin{tabular}{lrr}
\hline $\begin{array}{l}\text { Number } \\
\text { of sensor } \\
\text { positions }\end{array}$ & $\begin{array}{r}\text { Duration of scanning } \\
\text { at point densities of } \\
\text { approx.6 mm @ } 10 \mathrm{~m}\end{array}$ & $\begin{array}{r}\text { Total checking } \\
\text { time including } \\
\text { setting-up }\end{array}$ \\
\hline 5 & $10 \mathrm{~min}$ & $30 \mathrm{~min}$ \\
6 & $12 \mathrm{~min}$ & $35 \mathrm{~min}$ \\
\hline
\end{tabular}

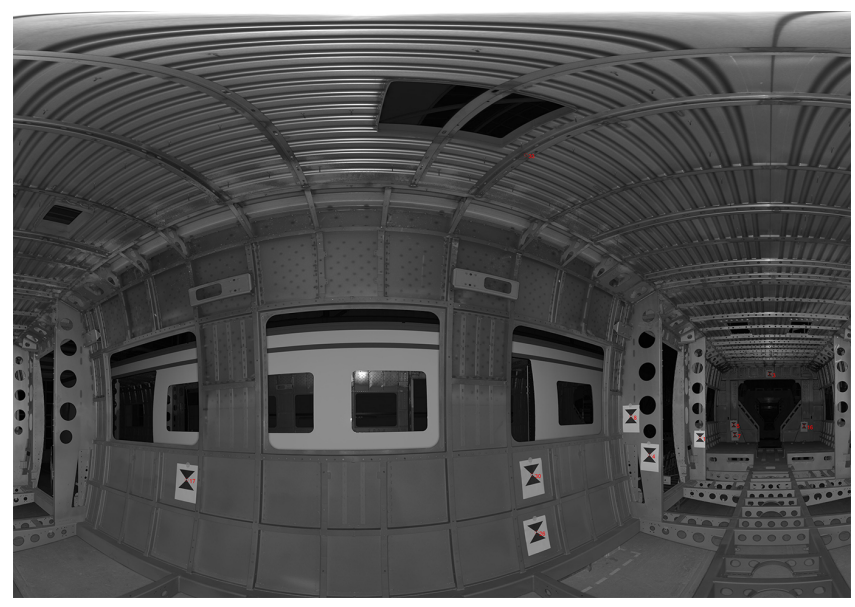

Figure 3. Example of a single point cloud: scanning result of one sensor position (of a total of five positions for this type of car body shell). The intensity of reflection (remission) is shown as a grey value, which results in a photo-realistic view of the point cloud. The targets are placed in a random pattern.

\section{Data analysis approach}

\subsection{Registration of point cloud data}

As scanning takes place from various sensor positions, first the individual scans should be registered and later summarized to one aligned (registered) general point cloud. Reference marks may be used to support the alignment (registration) of the different point clouds. Automated software is available for this as far as the targets are placed in a random pattern (Fig. 3 gives example for targets, placed in random

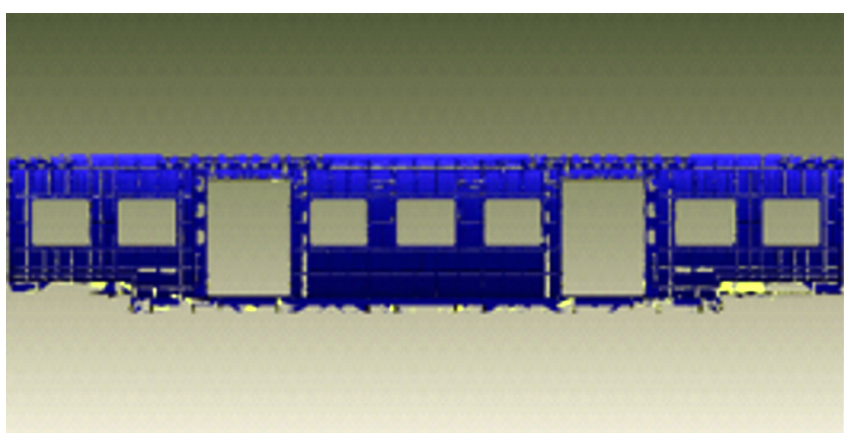

Figure 4. Point cloud from five sensor positions after registration and after meshing (software PolyWorks).

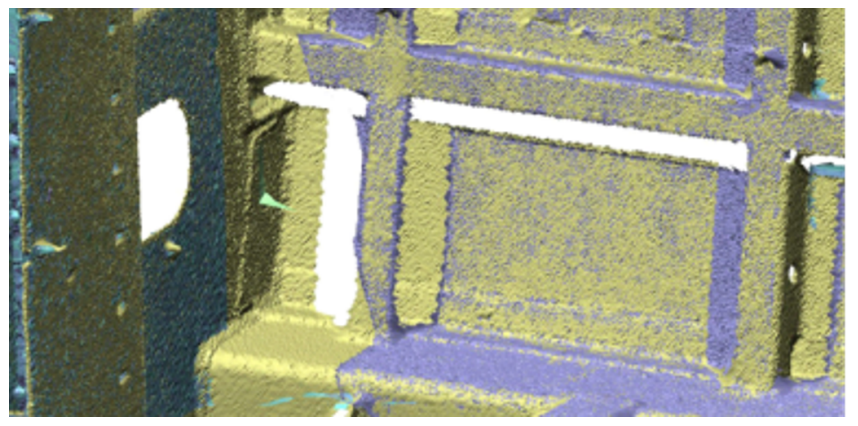

Figure 5. Best fit result for reducing the gaps of the scans from the different sensor positions (scan one: yellow; scan two: blue) (software PolyWorks).

pattern). For symmetrically placed targets, there is an option to name the targets. For summarizing different scans to an aligned general point cloud, meshing would be a suitable method because the points are reasonably thinned out and individual erroneous scans of the hall ceiling, for example, are eliminated. Figure 4 shows a meshed general point cloud from five sensor positions for a complete car body shell.

The point cloud thus obtained by the scanners and the subsequent processing represents a detailed image of the as-is state (actual state). Figure 5 shows a detail of the interior of the car body shell. The partial scans are well aligned with each other and interpenetrate each other. Minor shadowing is shown in white. 


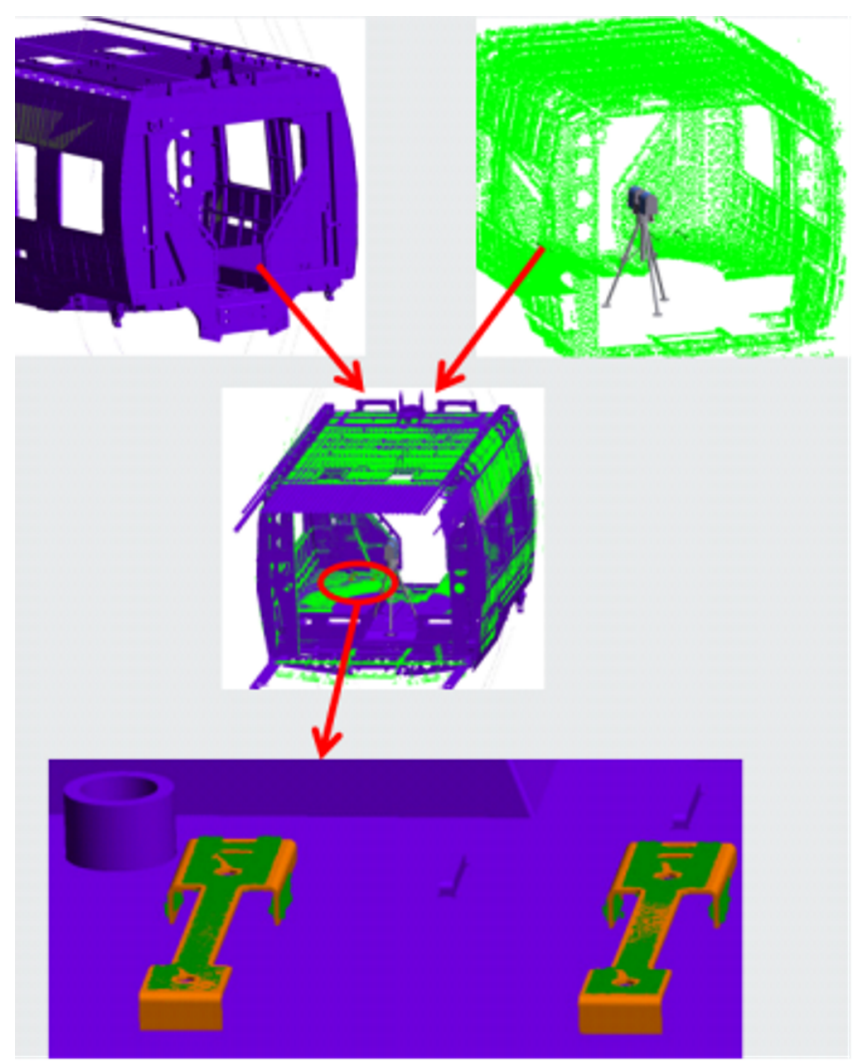

Figure 6. Section of a difference figure, two mounted parts, feature okay according to the CAD-point cloud comparison (software SpatialAnalyzer). The size of the mountings in the lower half of the figure is approx. $250 \times 50 \times 20 \mathrm{~mm}$.

The target state is installed by means of the 3-D design models, in the .catpart format, for example.

Comparisons between the scanned point cloud and the 3-D model may be performed by computing of difference figures.

These difference figures provide information about completeness. The difference figures are generated according to a newly developed algorithm. This algorithm connects the scope of functions of respective program packages and extends it. Software program packages for point cloud analysis and also for the generation of difference figures are already available. But first, the area-by-area consideration of both the 3-D model and the corresponding points of the point cloud were new.

Figure 6 illustrates the approach greatly simplified. First, it scans the car body shell inside. And later, it superposes the 3-D model in order to generate the difference figure. A small section of the colour-coded difference figure is also presented in Fig. 6, in the lower half of the figure. Exemplified by two mountings (on a much larger scale), the colour-coded distance between the 3-D model and the point cloud is shown.

\subsection{Tolerance specifications}

Here, two tolerance fields were considered:

i. the global tolerance field for the positioning of the mounted parts;

ii. and the local tolerance field for the positioning of the structural elements accommodating the mounted parts.

After all, the tolerance field of the car body shell is $\pm 14 \mathrm{~mm}$ based on a nominal length of $18 \mathrm{~m}$ according to Table 1, line B of DIN EN ISO 13920 (DIN EN ISO 13920:199611). It is therefore larger than or in the same range as the size of the mounted parts located on the rear wall. Thus, the complete scanned point cloud cannot be compared with the complete 3-D model at once. The comparison must rather be carried out area by area. For this purpose, it is differentiated between global coordinate system and local coordinate system. The coordinate systems can be superimposed, area by area, so that the local coordinate system is registered in the global one. In this way, the consideration of two coordinate systems with their tolerance fields can be simplified to just one tolerance field. Due to this superposition, the global tolerance field need not be considered any more (i.e. that of the car body shell length of $14 \mathrm{~mm}$ ), so that only the local tolerance field (i.e. that of the mounted parts) has to be considered. The consideration of the possible and accepted manufacturing tolerances of the nominal dimensions avoids the issue of second-type negative reports.

\subsection{Description of the new algorithm}

First, the point cloud of the 3-D scans is to be prepared, which means

i. registration (consolidation) of the individual scans of the different sensor positions

ii. and meshing.

Example parameters for meshing: mesh size of maximally $4 \mathrm{~mm}$ and single point deviation of $0.1 \mathrm{~mm}$. The points can optionally also be evaluated according to scanner distance and particularly to probing angle.

For the computing of difference figures, both the 3-D model and the general point cloud were divided into sections. To compare the complete 3-D model with the complete general point cloud would lead to the following. The deviation of the real car body shell to the 3-D model would increase the difference between the 3-D model and the point cloud. To divide into areas allows one to fit the point cloud closer to the 3-D model.

The best fit is to overlay the 3-D model with the general point cloud. But this is to be done just in the investigated area of the car body shell. To find the solution of the inspection task discussed here, the possibility of performing, besides best fit, an iterative orientation of the point cloud against 
CAD by means of relationships is also important (compensation with constraints of defined relationships, e.g. RPS or limitations, only in certain axial directions).

Another possibility, in addition to the best fit, is the RANSAC algorithm. The RANSAC algorithm is often used to make best fits robust against outliers. For this purpose, equalization is performed with a strongly reduced point number, and the obtained parameters are used for the remaining points. On the one hand, this may reduce the necessary calculation time and, on the other hand, which is even more important, it may prevent outlier points from disturbing the best fit, thus impeding the finding of the outliers according to the residuum criterion.

Then, area by area, the following algorithm is applied.

i. A distance $\varepsilon$ is defined within which the point cloud is considered in relation to the 3-D model.

ii. Now all elements of the 3-D model are checked for whether there are corresponding points (distance smaller $\varepsilon$ ) in the point cloud. For this purpose, not only is distance used as a search criterion, but an angle examination is also carried out. It can optionally be adjusted so that a comparison is made with the highest CAD element, i.e. neither interior CAD elements nor those oriented against the scan points are used, which minimizes error susceptibility, particularly for complex CAD assemblies (see also Sect. 5.1).

iii. The CAD elements with corresponding points in the point cloud are marked.

iv. The marked elements should be present and are present.

v. In the next step the selection of the elements of the 3D model is reversed/inverted. Now all 3-D model elements having no correspondence in the point cloud are marked.

vi. These elements should be present but are not present, according to the point cloud.

vii. The selection of 3-D model elements therefore exactly shows those possibly missing, being positioned wrongly, i.e. being at least suspect and requiring another consideration/manual recheck.

The hypothesis for these selected criteria: not present or positioned outside the tolerated limits.

A manual verification can be done easily and sufficiently fast as far as the number of test criteria has been reduced from several hundreds to much less than 100 by means of the workflow described here. Figure 7 shows a respective analysis for a sidewall area. At comparatively many places in Fig. 7 this distance between the 3-D model and the general point cloud is larger than $\varepsilon$, which is also visible here in the colour marking. Thus, the noise also becomes apparent

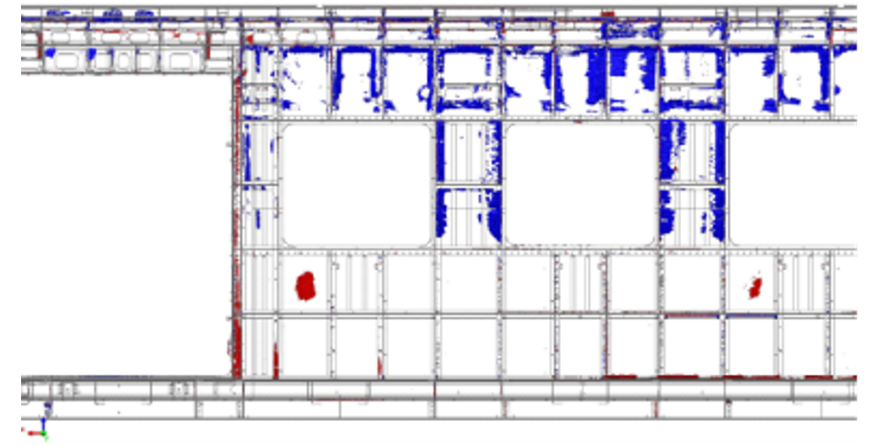

Figure 7. Sidewall detail inside. Presentation of the 3-D data set. The colour marking codes the distance of the corresponding points of the point cloud (software PolyWorks). The value for $\varepsilon$ is $3 \mathrm{~mm}$. Red and blue express \pm .

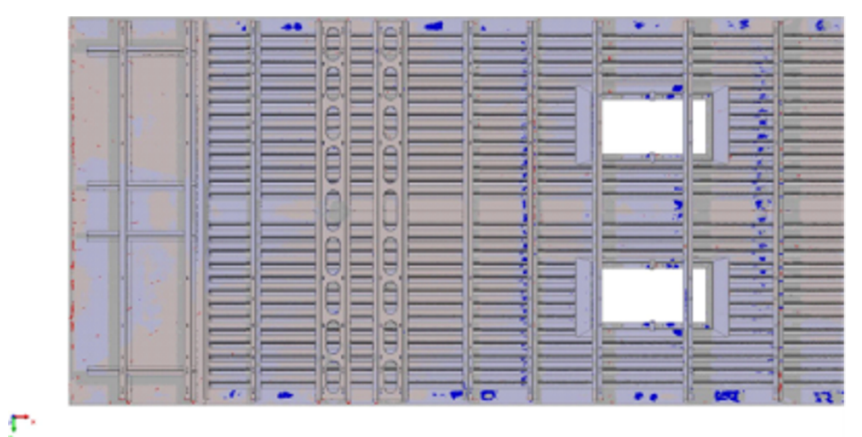

Figure 8. Roof segment area inside. Presentation of the 3-D data set. The colour marking codes the distance of the corresponding points of the point cloud (software PolyWorks). The value for $\varepsilon$ is $3 \mathrm{~mm}$. Red and blue express \pm .

in the capture of the point cloud and can only partially be compensated for by the algorithm.

The defined value for $\varepsilon$, as well as the measurement noise, define the display quality of the difference figure.

The application of the algorithm to the roof in Fig. 8 shows a much sharper result. Various capture parameters are partially responsible for that. At only a few points is the distance of the point cloud to the 3-D data set larger than $\varepsilon$. A few small points therefore become visible in the colour marking. The analysis of the difference figure already leads to the following statement: no mounted part is occupied with such low density with points that it must be assumed to be missing.

The application of the algorithm to the rear wall in Fig. 9 also shows a sufficiently sharp result. At only a few points is the distance of the point cloud to the 3-D data set larger than $\varepsilon$. A few small points therefore become visible in the colour marking. An experienced inspector would already recognize in view of the difference figure that no mounted part is occupied so densely with missing points that it must be assumed to be missing. 


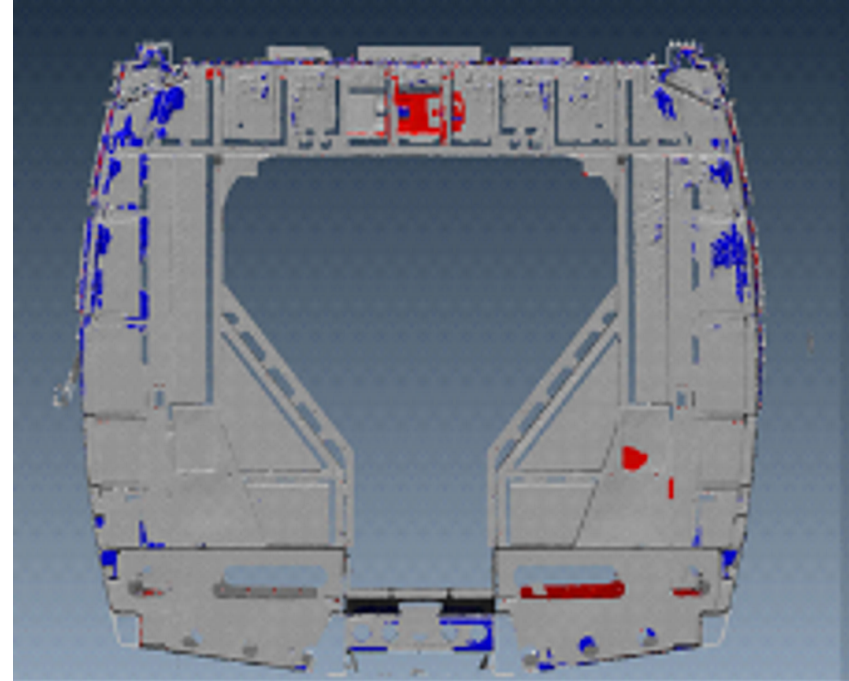

Figure 9. Rear wall area. Presentation of the 3-D data set. The colour marking codes the distance of the corresponding points of the point cloud (software PolyWorks). The value for $\varepsilon$ is $3 \mathrm{~mm}$. Red and blue express \pm .

\section{Performance and accuracy}

The presented approach for the completeness check is partly new. The inspected construction is divided into areas. And the computing of the difference figures is performed area by area, after best fit just inside the area. This allows one to handle the basic problem that the real length of the car body shell only complies with the nominal size from the CAD data set within the tolerances of several millimetres. The solution therefore is to fit individual areas of the point cloud iteratively to CAD and to subsequently compare each point cloud segment to CAD.

\subsection{Limiting factors}

The filter setting for so-called mixed points is of importance. When the TLS/HDS laser beam hits an edge and is reflected by this edge as well as possibly by the surface behind it, this is called a "mixed point". For short distances from the edge to the surface behind it, the mixed point cannot always be recognized reliably, and so is not excluded from further analysis. The smaller the diameter of the laser beam, the smaller the probability that it will hit an edge. A smaller diameter of the laser beam will therefore result in fewer mixed points. Table 1 lists the diameters and data of the beam geometry of a typical TLS. An infinitely small beam diameter can no longer be divided by any edge, which, however, cannot be demonstrated technically.

It must be clarified whether the contour of the confidence interval of probing (the anisotropic uncertainty portion of the measured value) is to be considered as an impact on further analysis of the measured point coordinates of the point

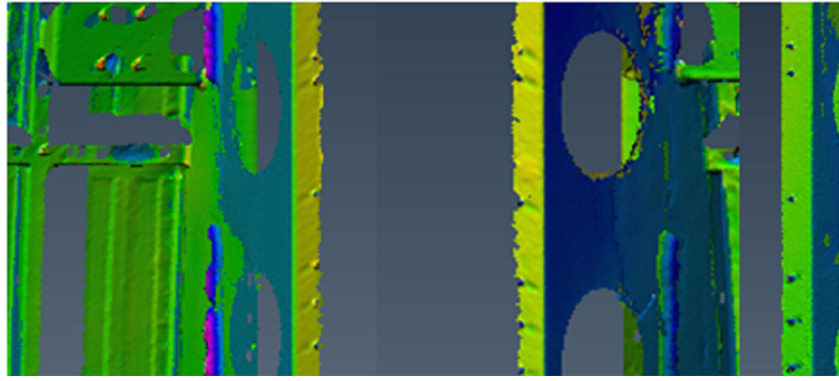

Figure 10. Sidewall detail, meshed point cloud (software PolyWorks). For the scale, please refer to Fig. 15

clouds. In the case of inclined incidence of the laser beam on the surface, the measured value itself does not change considerably. Although the laser circle becomes an ellipse on the surface, the "centre of the remission spot" does not change. In the case of very inclined incidence, less light is scattered back and measurement noise increases. In order to limit this effect and therefore the noise, a limit value for the probing angle was set in the filter settings and flatter probing was hidden. The limit value in the filter amounts to between 45 and $57^{\circ}$.

\subsubsection{On the edge problem}

The probing of surfaces is performed point by point using an adjustable distance between the points.

With the yellow stripe in the centre, Figure 10 shows a flange scanned in a preliminary test. The holes seem not to be completely surrounded by material (notches instead of completely surrounded holes). In fact, there were just no scanned points any more between the holes and the flange edge, which illustrates the requirement of selecting a sufficiently small point distance $p$ in the scanner parameters. The green stripe on the right-hand side of Fig. 10 provides a better view of reality. The holes are surrounded by material which has also been probed. The point-by-point probing therefore leads to the fact that the real edge runs in-between two probings and cannot be specified more exactly than the point distance.

Figure 11 demonstrates another problem. The crossbar presented as completely missing (in blue) is actually present and included in the point cloud. The presentation of a deviation results from the comparison between the 3-D model data and the point cloud. It makes a difference whether the "closest" surface of the data set or the "highest" surface of the 3-D model is to be used for analysing. Correct orientation within the data set is therefore a prerequisite too.

Figures 12 and 13 were presented to visualize the difference between "closest" nominal surface and "highest" nominal surface.

Figure 12 shows, in the upper half of the picture, points that refer to the visible surface. Points in the right half of the 


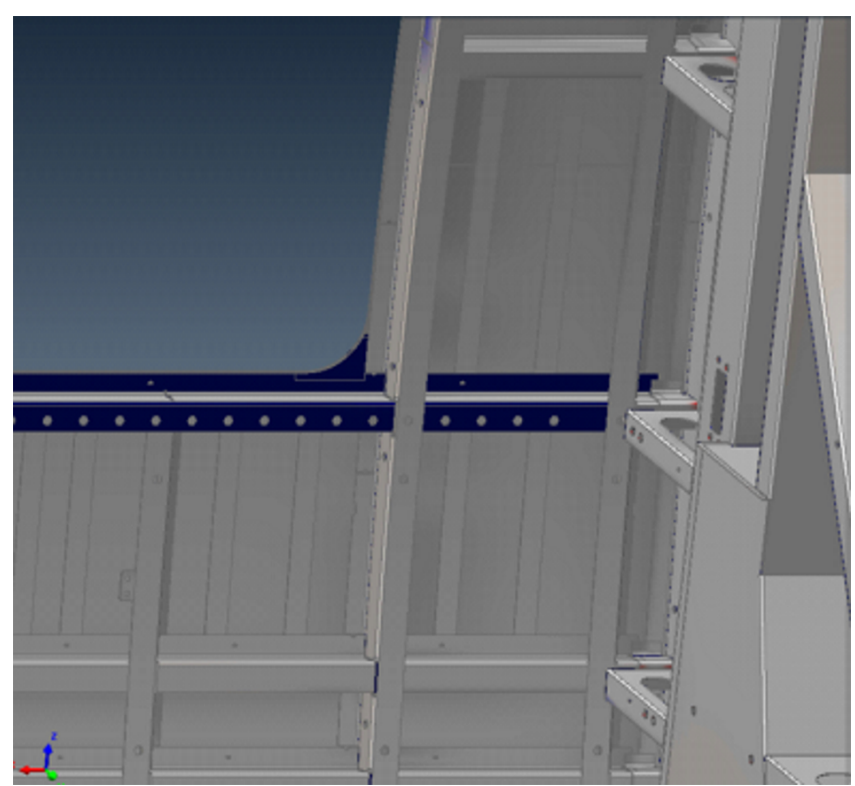

Figure 11. Sidewall segment detail close to the rear wall. Presentation of the 3-D data set. The colour marking codes the distance of the corresponding points of the point cloud (software PolyWorks). The value for $\varepsilon$ is $3 \mathrm{~mm}$. Red and blue express \pm .

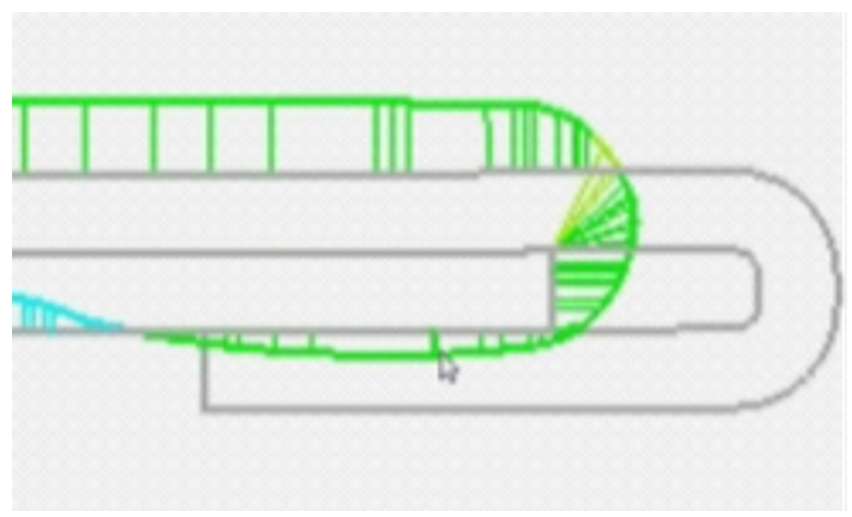

Figure 12. Points in relation to the "closest" nominal surface (software PolyWorks).

picture are compared with the invisible surface, instead of being compared with the visible surface.

But this invisible surface is the closest one. Even the points in the lower half of the picture are in relation to the closest surface, not to the visible one.

Figure 13 shows points in relation to the highest surface, the visible surface. Just the yellow marked relations are between points and the closest surface (it seems to be a bug).

In Figs. 12 and 13, the points in the right half of the figure are relevant to get the discrepancy. The marked distance to the closest surface in Fig. 12 (green) is smaller than the marked distance to the highest surface in Fig. 13 (dark blue

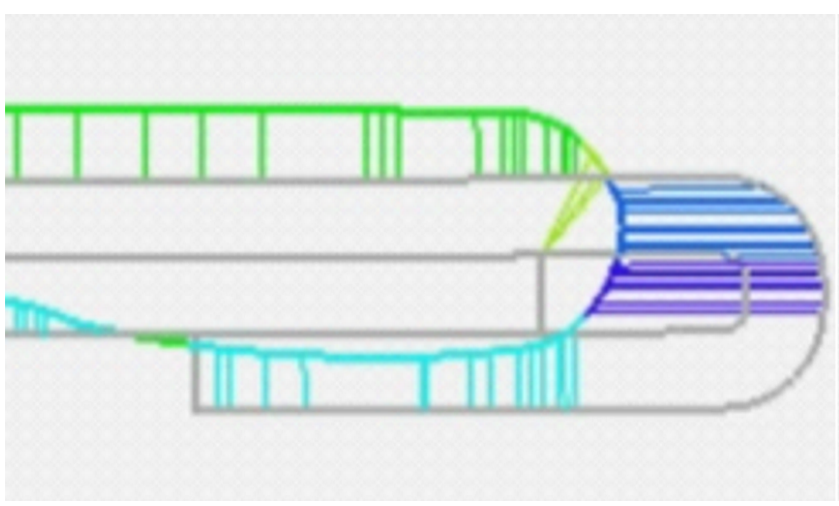

Figure 13. Points in relation to the "highest" nominal surface (software PolyWorks).

and violet). And because the distance is different, implications therefrom may differ.

\subsection{Achieved accuracy}

The point cloud is captured by the phase shift scanner with accuracies to within some tenth $(1 / 10)$ of a millimetre. A standard deviation of $0.3 \mathrm{~mm}$ and a maximum deviation of a single point of $0.7 \mathrm{~mm}$ are indicated for the subsequent registration by means of automatically detected target marks. This provides good data for meshing.

Whereas Fig. 4 shows the meshed point cloud of the whole car body shell (scanned from inside), Fig. 14 demonstrates a virtual cross section. The colour marking shows the distance of the corresponding points of the point cloud to the CAD model (distance CAD model to point cloud).

For a better orientation in the following figures, Fig. 15 shows a detail of Fig. 14. Where the colour scale changes from blue to green, there is zero deviation between the 3-D model and the mesh from the general point cloud (see the scale in Fig. 15). The full-surface colouring in Figs. 14 and 15 does not yet result in a sharp display of the completeness of the mounted parts.

For this reason, the colour scale of the difference figure was changed for Figs. 7, 8 and 9. Just elements with point distances larger than $\varepsilon$ are colour-marked in Figs. 7, 8 and 9. This colour scheme was also applied in Fig. 11.

A look at the side wall near the rear wall and at the rear wall itself shows red points in Fig. 16. These are protective plugs of plastic which had already been mounted at the time of scanning and which do not belong to the design data set of the car body shell, of course. The detection of these $\varnothing 8 \times 20 \mathrm{~mm}$ and $\varnothing 10 \times 20 \mathrm{~mm}$ large criteria demonstrates the potential sensitivity of the test system. Differences between the 3-D model and the point cloud are made detectable and visible by the application of the algorithm. Even such small criteria as the protection pins are therewith captured and analysed. 


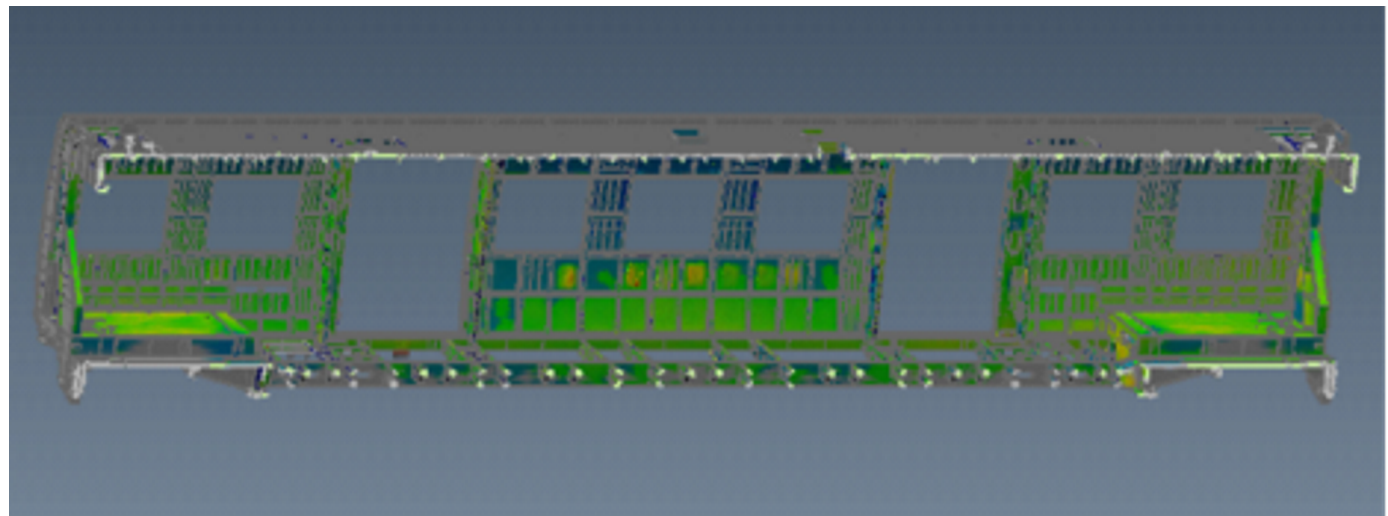

Figure 14. Virtual cross section through the whole car body shell. The colour marking shows the distance of the corresponding points of the point cloud (software PolyWorks). For the scale, please refer to Fig. 15.

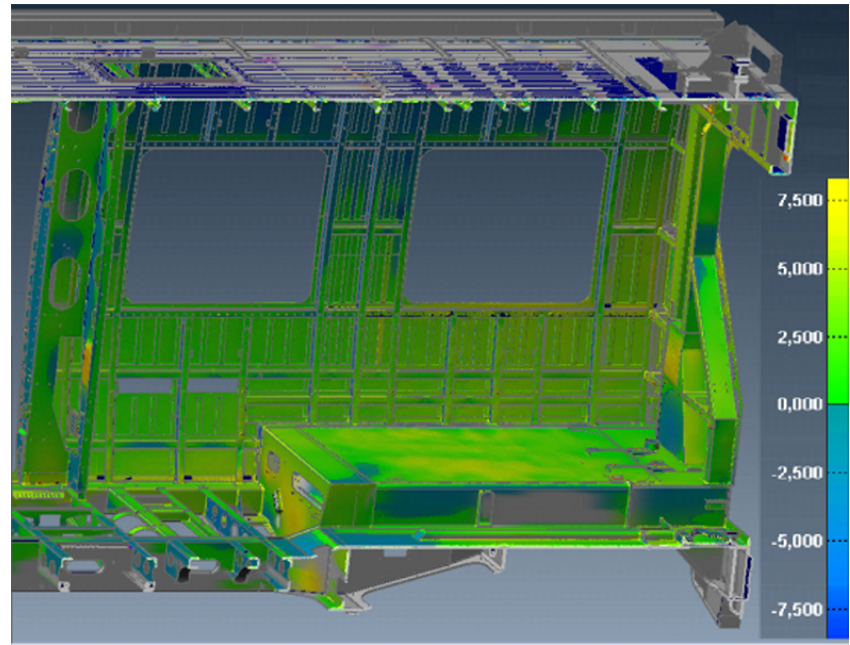

Figure 15. Detail of Fig. 14.

Figure 17 shows the grey-value image of the point cloud directly after scanning so as to compare it with the colourcoded 3-D model images. The plastic plugs are depicted in sharp outlines but with less contrast.

Moreover, Fig. 17 shows one of the automatically detected and numbered target marks. By using these target marks, the registration of the individual point clouds from the different sensor positions took place so as to obtain the general registered point cloud. Table 3 shows some criteria, which were detected by scanning and recognized in the following algorithm. The flaps as well as the protection pins were explicitly rendered in the difference figure (Fig. 16).

\section{Conclusion and outlook}

The developed solution for completeness checking was approved for mountings at car body shells.

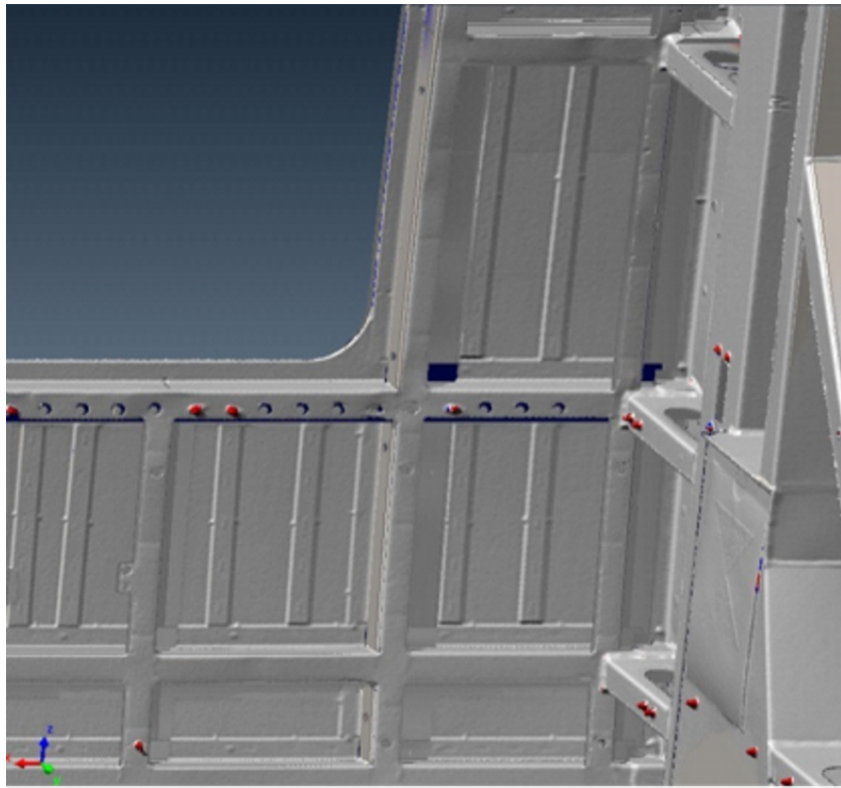

Figure 16. Sidewall segment detail of the rear wall. Presentation of the 3-D data set. The colour marking codes the distance of the corresponding points of the point cloud (software PolyWorks). The value for $\varepsilon$ is $3 \mathrm{~mm}$. Red and blue express \pm .

The application limits of test processes (data acquisition) known so far and the limits of software-side further processing of information (i.e. post-processing) were extended so that now an additional inspection task in manufacturing practice can be carried out by equipment support (Jurdeczka, 2016b, c).

The issue of difference figures with missing parts marked therein therefore allows completeness checking that is largely independent of the inspector's tiredness and experience.

The noise behaviour can be influenced by adjustment so that a safe detection of criteria of typically 
Table 3. Scanning achievements.

\begin{tabular}{lll}
\hline & Surphaser 100HSX-SR & ScanStation P40 \\
\hline Point distances for scanning & 30 LPD & $6.3 \mathrm{~mm} @ 10 \mathrm{~m}$ \\
Alignment statistics: single scans to & Standard deviation of $0.3 \mathrm{~mm}$ and a maximum & Currently not \\
the general point cloud (41 targets) & deviation of a single point of $0.7 \mathrm{~mm}$ & be determined \\
Detected criteria & Protection pins $\varnothing 10 \times 20 \mathrm{~mm}$ & Flaps $15 \mathrm{~mm} \times 25 \mathrm{~mm} \times 1 \mathrm{~mm}$
\end{tabular}

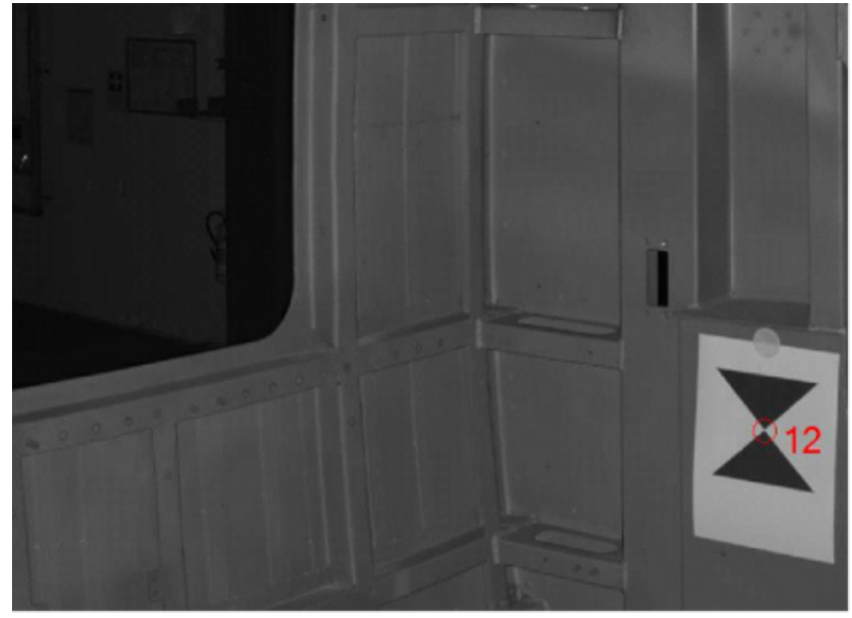

Figure 17. Grey-value image directly after scanning, detail of a point cloud, comparable with Fig. 3, and an automatically detected and numbered target mark can also be recognized.

$15 \mathrm{~mm} \times 25 \mathrm{~mm} \times 1 \mathrm{~mm}$ can be achieved. The generation of difference figures according to the presented algorithm very reliably provides evidence of defaults (missing mounted parts, clear fault positions). Please also refer to the comparison between Figs. 7 and 8 and 9.

The conclusion statement of the equipment-based test system considerably supports the inspector's work of completeness checking.

The test system has already been used in a cyclic production line for testing purposes. The results are very promising. For this reason, an innovation project was launched by Alstom and a cooperation agreement was concluded with the Institute for Production Metrology of the Technical University of Braunschweig.

The scope of the test procedure described here can be stated as a car body shell for railway vehicles with main dimensions of $18 \mathrm{~m} \times 2.5 \mathrm{~m} \times 2.5 \mathrm{~m}$ with a typical tolerance zone of $14 \mathrm{~mm}$ and mountings on it with a typical dimension of $15 \mathrm{~mm} \times 25 \mathrm{~mm} \times 1 \mathrm{~mm}$. It is to be applied to other steel constructions, manufactured according to DIN EN ISO 13920 (DIN EN ISO 13920:1996-11), too.

Borders are given by scan accuracy, the quality of the following alignment of several single point clouds and by the measurement range of the 3-D scan. Even the point distance, which is to be realized, limits the smallness of inspected cri- teria. The scan accuracy is a function of scan distance. A substantial discussion of the accuracy of TLS is given by Wunderlich et al. (2013). As shown, the distance between two scan points should be smaller than $\varepsilon$. And referring to Fig. 7, even the noise should be smaller than $\varepsilon$. Both limit the size of checked criteria to not smaller than $2 \mathrm{~mm}$.

For car body shells, this is given. Many of the approx. 300 mounted parts are recognized as being correct and present. Detected defaults are presented in the model in a traceable and clearly visible way. Manual rechecking of these comparatively few places then separates real fault displays from occasional faults of higher order.

As the target description is done by means of a 3-D CAD data model, the test method can also be classified as modelbased.

The model-based target description leads to high flexibility in changes to the test objects (car body shells) and is therefore especially suitable for varied assembly processes (Berndt and Warnemünde, 2016). Constructional changes in the CAD model can therefore also be integrated into the test plan (list of criteria to be checked) in a semi-automated way. The structure of the 3-D model data are of importance for the scope of the calculation operations. As far as it is possible by reasonable structuring to issue a so-called light model with mounted parts separate from the complete design data set, suitable input information about the target state will be available. The light model can be considered as an extract from the parts list, i.e. it lists all mounted parts. The import and export of test criteria plans of the light model can then be supported very well. Thus, test criteria plans can largely be automated (name of the mounted part, identification number, etc.).

A classification of the test object in areas, both in the data set and in the point cloud, should become part of the testing process. For these geometric areas, optimum geometric area sizes should be determined to obtain minimum noise. Additional investigations are required here (Jurdeczka, 2016d).

Completeness checking cannot only be documented visually, but also statistically. As far as scripts can be generated, the processes should also be automated in the software.

While there is no subroutine for presenting the results, slides in isometric projection can be the useful way to present the difference figures, or slides in dimetric projection. Figures 16 and 17 are examples thereof. 


\section{Data availability}

For the completeness check, in the way described here, a 3D model as well as point clouds are necessary. These data were provided by Alstom, for the development of the test procedure. Since there is design information contained in the data, it is shown here in a generalized way.

Competing interests. The author declares that he has no conflict of interest.

Acknowledgements. This study was supported by the institutes/companies mentioned here and in the text above. I would like to express many thanks for their support so far.

Edited by: H. Bosse

Reviewed by: two anonymous referees

\section{References}

Berndt, D. and Sauer, S.: Automatic Completeness Check: Optical Measurement Technologies Detect the Smallest of Errors, Press Release of Fraunhofer Institute for Factory Operation and Automation IFF, Magdeburg, 5 May 2014, available at: http://www.iff.fraunhofer.de/content/dam/iff/en/documents/presskit/, (last access: 22 December 2016), 2014.

Berndt, D. and Warnemünde, R.: Flexible modellbasierte Montageprüfung, Prüfung durch Vergleich mit synthetischen Messdaten, available at: http://www.iff.fraunhofer. de/de/geschaeftsbereiche/messtechnik-prueftechnik/ montagepruefung-modellbasiert.html (last access: 5 December 2016), 2012.

Berndt, D. and Warnemünde, R.: Flexible model based assembly inspection, available at: http://www.iff. fraunhofer.de/content/dam/iff/en/documents/publications/ flexible-model-based-assembly-inspection-fraunhofer-iff.pdf, last access: 5 December 2016.

Demant, Ch., Streicher-Abel, B., and Garnica, C.: Industrial Image Processing, Visual Quality Control in Manufacturing, 2. Auflage, Springer Heidelberg Dordrecht London New York, 265301, 2012.

DIN EN ISO 13920:1996-11: Schweißen - Allgemeintoleranzen für Schweißkonstruktionen - Längen- und Winkelmaße; Form und Lage (ISO 13920:1996); Beuth Verlag Berlin, Welding General tolerances for welded constructions - Dimensions for lengths and angles; shape and position (ISO 13920:1996), German version EN ISO 13920:1996, 1996.

Drechsel, T.: Laserprojektion im Lokomotivbau. Positionieren von Anbauteilen in der Produktion der Lokomotive Vectron, DVV Media Group/Eurailpress, EI-Eisenbahningenieur Jahrgang 67, 4, p. 16, 2016.
Extend3d GmbH: Industrial laser and video projection for more efficient processes, available at: http://www.extend3d.de/en/ anwendungen.php?content $=$ schweissen, last access: 5 December 2016.

Jurdeczka, U.: Analyse von Wagenkästen für Schienenfahrzeuge mit PolyWorks Inspector Premium, Presentation at 15th, PolyWorks User Meeting of the Duwe-3d AG, 6-8 April 2016 in Friedrichshafen, 2016a.

Jurdeczka, U.: Optisches Prüfsystem für variantenreiche Montageprozesse, Carl-Hanser Verlag München, QZ Jahrgang 61, 7, p. 46, available at: http://www.qz-online.de/ (last access 27 January 2017), 2016 b.

Jurdeczka, U.: Konzeption zur Vollständigkeitsprüfung von gefügten Strukturen am Beispiel Schienenfahrzeugwagenkästen, Posterpresentation at the 18th GMA/ITGSymposium Sensors and Measurement Systems 2016, Nürnberg, doi:10.5162/sensoren2016/P7.8, 2016c.

Jurdeczka, U.: Analyse an Schienenfahrzeug-Wagenkästen mit Hilfe von 3D Scans und einem angepaßten Algorithmus für die Erzeugung von Differenzbildern, Presentation at the 19th Application-oriented Workshop on Measuring, Modeling, Processing and Analysis of 3D-Data, Berlin-Adlershof, December 2016, ISBN 978-3-942709-16-3, 2016d.

Kelch, J.: Vollautomatisierte 100\% Inspektion von KarosserieteilenAus allen Perspektiven, Carl-Hanser Verlag München, QZ Jahrgang 60,05 , p. 62 , available at: http://www.qz-online.de/ 974021 (last access 27 January 2017), 2015.

Optimum datamanagement solutions GmbH: Der "Schlaue Klaus" Anwendungen für intelligente Bilderkennung. Optimum GmbH, Karlsruhe, available at: https://www.optimum-gmbh.de/ der-schlaue-klaus.html, last access: 15 December 2016.

Reiterer, A., Höfler, H., and Wölfelschneider, H.: Aufbau und Funktionsprinzip mobiler Laserscanner - Stand der Technik und neueste Trends, in: Beiträge zum 147, DVW-Seminar in Fulda, Schriftenreihe des DVW, Band 81/2015, p. 27, 2015.

Stemmer, H.: Bevor Fehler entstehen, 3D Bildverarbeitungssystem prüft Schaumstoffteile, Carl-Hanser Verlag München, QZ Jahrgang 60,11 , p. 64; available at: http://www.qz-online.de/ 1047972, 2015

Störig, T. and Zimmermann, R.: How we use nine LEICA Ultra High Speed Scanners to ensure construction quality on an efficient cruise ship factory line, Proccedings of HxGN Live Conference, Las Vegas, 2 June 2015, 20-25, 2015.

Tarih, C.: Aktueller Stand der Automatisierung von Sichtprüfungen, speziell Vollständigkeitsprüfungen an großen Stahlbaustrukturen am Beispiel Schienenfahrzeugwagenkästen, Seminarpaper at Ostfalia University of Applied Sciences Braunschweig/Wolfenbüttel, Faculty Mechanical Engineering, 2016.

Wunderlich, T., Wasmeier, P., Ohlmann-Lauber, J., Schäfer, T., and Reidl, F.: Objektivierung von Spezifikationen Terrestrischer Laserscanner - Ein Beitrag des Geodätischen Prüflabors der Technischen Universität München, Blaue Reihe des Lehrstuhls für Geodäsie, Heft 20, 02, 8-19, 2013. 